\title{
Kulturen der Kontrolle: Zum Risiko- und Konfliktmanagement des modernen Rechtsstaates
}

\section{Thematische Einführung}

Moderne Staaten sind Ordnungen garantierter Sicherheit und d.h. wirkungsmächtiger Ausdruck eines gelungenen Säkularisationsprozesses. ${ }^{1}$ Darin eingeschrieben ist eine Entwicklung, die wir heute als ebenso problematische wie notwendige Aufklärungsgeschichte der Vernunft beschreiben können. Letztere hat nicht nur zu der inzwischen unbestrittenen und ordnungspolitisch wichtigen Unterscheidung von bürgerlicher Gesellschaft und Staat, sondern auch zu einer rechtlichen Emanzipation der handelnden Akteure geführt. Entscheidend ist aber, dass mit der genannten (Rechts-)Sicherheitsgarantie zugleich die Unanfechtbarkeit der hoheitlichen Macht behauptet wird; eine $\mathrm{Au}$ torisierungsstrategie, die sie zu einer fundamentalen Bedingung von Gesellschaft und Staat werden lässt. ${ }^{2}$ Die Einschätzungen darüber, worauf diese Semantik der Ordnung zielt und welche normativen oder auch systemischen Implikationen damit verbunden sind, gehen jedoch weit auseinander. Eine Ausnahme bildet der Vorbeuge- und Präventionsgedanke. ${ }^{3}$ Er findet sich in Konzepten mit unterschiedlichster struktureller und institutioneller Ausrichtung und genießt dabei ein kaum zu übertreffendes Maß an Attraktivität. „Dass es sinnvoller ist, künftige Übel durch geeignete Interventionen zu vermeiden, als sie erst dann zu bekämpfen, wenn sie manifest geworden sind, das erscheint uns so selbstverständlich, dass es keiner weiteren Begründung bedarf. “4 Nun wäre es eine durchaus interessante Aufgabe, sich den unterschiedlichen normativen, aber auch systemischen Implikationen gegenwärtiger Ordnungs- und Staatsmodelle zu widmen. Indes, um einiges reizvoller scheint es zu sein, sich einem Phänomen, wie der „Interventions-Prävention“ zu nähern, das in den Kontexten moderner Staatlichkeit kaum oder nur oberflächlich diskutiert und noch weniger in Zweifel gezogen wird. Gerade dieser Selbstverständlichkeit und Omnipräsenz eines gesellschaftlichen und insofern auch rechtlichen Deutungsmusters soll im Folgenden auf den Grund gegangen werden. Anliegen der Erörterungen ist es, nach Sinn und Tragweite des interventionsbasierten Risiko- und Konfliktmanagements des modernen Staates zu fragen, dessen Bedeutung für

1 In diesem Sinne bereits M. Weber, Wirtschaft und Gesellschaft, Tübingen 1985; zur aktuellen Debatte etwa A. Anter, Die Macht der Ordnung, Tübingen 2007; E.-W. Böckenförde, Der säkularisierte Staat, München 2007 und C. Taylor, Ein säkulares Zeitalter, Frankfurt a.M. 2009.

2 Instruktiv dazu P.-A. Albrecht, Der Weg in die Sicherheitsgesellschaft, Berlin 2010; darüber hinaus G. Agamben, Ausnahmezustand, 2004; M. Foucault, Analytik der Macht, Frankfurt a.M. 2005; N. Luhmann, Macht, Stuttgart 1975; M. Möstl, Die staatliche Garantie für die öffentliche Sicherheit und Ordnung, Tübingen 2002; H. Popitz, Prozesse der Machtbildung, Tübingen 1976; C. Schmitt, Der Begriff des Politischen, Berlin 1963 und P.-T. Stoll, Sicherheit als Aufgabe von Staat und Gesellschaft, Tübingen 2003; R. Wahl, Prävention und Vorsorge, Bonn 1995.

3 Siehe dazu etwas F. Ewald, Der Vorsorgestaat, Frankfurt a.M. 1993 und S. Huster (Hrsg.), Vom Rechts- zum Präventionsstaat, Frankfurt a.M. 2008; weitere Literaturnachweise folgen im Text.

4 So U. Bröckling, in: Behemot. A Journal on Civilisation, 2008 (1), S. $38 \mathrm{ff}$. 
die moderne (Straf-)Rechtspraxis zu analysieren und so die Tiefenstrukturen der entsprechenden Argumente zu beleuchten. In einem ersten Schritt soll deshalb die Genealogie und Struktur des Rechtsgewährleistungsparadigmas näher in den Blick genommen werden (II). Ein zweites Kapitel wird sich den zentralen Strategien gegenwärtiger Risiko- und Konfliktanalyse widmen (III). Der dritte Abschnitt will wiederum zeigen, welche Logik den modernen Sicherheitsgarantien zugrunde liegt und welche Probleme damit einhergehen (IV). Der Beitrag endet mit einer Zusammenfassung der wichtigsten Einsichten (V).

\section{Die ordnungspolitische Matrix von Leistung und Legitimation}

\section{Sicherheitsverlangen und Sicherheitsversprechen}

Die Rede von einer säkularen Ordnungsmacht, von einem rationalisierten Sicherheitsversprechen oder ähnlichem verweist zunächst auf das einer rechtlichen Verständigung zugrunde liegende Leistungs-Legitmations-Paradigma. ${ }^{5}$ Danach verpflichten sich Staat und Gesellschaft, die elementaren Rechte resp. die Subsistenzbedingungen des Einzelnen, so effektiv wie möglich zu schützen. Im Gegenzug erkennen letztere das Gewaltund Justizmonopol hoheitlicher Institutionen an. ${ }^{6}$ Betont wird insofern ein Bezugsnetz aus freiheitsgenormten Anerkennungsstrategien und darauf beruhenden Funktionseinheiten, deren vorrangige Aufgabe es ist, die exzentrische Position des Einzelnen mit derjenigen der Allgemeinheit im Gleichgewicht zu halten. Dem bereits erwähnten Sicherheitsversprechen korrespondiert also immer ein darauf bezogenes Sicherheitsverlangen. Konflikte oder das, was wir als Konfliktformen definieren, machen allerdings deutlich, dass dieses Gleichgewicht zugunsten partikularer Geltungsbehauptungen durchbrochen, jedenfalls aber marginalisiert werden kann. Zur Disposition gestellt wird so nicht nur die ordnungspolitische Matrix, sondern auch das damit verbundene Normstabilisierungspotential. Wie eine Gesellschaft und d.h. eine autorisierte Ordnungsmacht darauf reagiert, hängt wesentlich davon ab, welche normativen Deutungs- und strukturellen Verarbeitungsmuster ihr im Einzelnen zur Verfügung stehen. Augenscheinlich wird dann aber auch, dass das Recht als originäres Konfliktlösungsverfahren zugleich den Charakter einer umfassenden Disziplinierungs- und Befriedungspraxis erhält. ${ }^{7}$

5 Zur Klärung der begrifflichen Grundlagen vgl. die Überlegungen des Verf., in: ZStW 120 (2008), $68 \mathrm{ff}$.

6 Zur aktuellen Diskussion des Gewalt- und Justizmonopols vgl. nur U. Di Fabio, Das Recht offener Staaten, Tübingen 1998; H. J. Faller, in: ders. (Hrsg.), Festschrift für Geiger, Tübingen 1989, 3; D. Merten, Rechtsstaat und Gewaltmonopol, Tübingen 1975, S. 29 ff.; H. Quaritsch, Staat und Souveränität, Bd. 1 Grundlagen, Frankfurt a.M. 1970 und bereits C. Schmitt, Verfassungslehre, München 1928; zur Genese des damit verbundenen Selbstverständnisses R. Koselleck, Kritik und Krise, Frankfurt a.M. 1973, S. 11 ff. und Weber (Fn. 1), S. 29 f.; aus kulturgeschichtlicher Perspektive N. Elias, Studien über die Deutschen, Frankfurt a.M. 1994, S. $282 \mathrm{ff}$.

7 Hier sind gegenwärtig eine Vielzahl von (Konfliktlösungs-)Praxen denkbar, neben der Mediation als spezifische Rechtsgestaltungsform, auch zivilrechtliche oder eben auch verwaltungsund strafrechtliche Verfahren; auf letztere wird sich der Fokus weitgehend beschränken. 


\section{Zur Genealogie des Rechtsgewährleistungsparadigmas}

Die begriffsgeschichtliche Referenz dieser so verstandenen Gewährleistungsmatrix bildet das staats- und souveränitätstheoretische Modell von Thomas Hobbes. Im Anschluss an Newton, Descartes und Galilei, um hier nur einige zu nennen, hat er gerade durch die Radikalisierung seiner philosophischen und epistemologischen Methode Wegweisendes geleistet. ${ }^{8}$

Diese „staatstheoretische Neujustierung“ oder Radikalisierung geht mit einem gewandelten Wissens- und Wissenschaftsverständnis einher. ${ }^{9}$ War die Wissensanalyse bzw. -vermittlung in der Scholastik und im ausgehenden Mittelalter noch Teil einer als kohärent behaupteten Heilsordnung, Glaube (Confessio/Religio) und Welterkenntnis (mundus sensibilis atque intelligibilis) also unmittelbar aufeinander bezogen, so wurde dieser Funktionszusammenhang zunehmend in Frage gestellt. ${ }^{10}$ Ähnlich wie Bacon und Kepler vertrat Hobbes die Überzeugung, dass sich Wissensressourcen - stricto sensu - nur im Kontext einer systematischen Wirklichkeitswissenschaft i.S. einer scientia propter potentiam verhandeln ließen, man denke nur an das novum organum oder die astronomia nova; Aussagen über die Religion, Gott oder entsprechende Heilserwartungen dagegen Fragen des Glaubens waren. ${ }^{11}$ Damit sollten weder Theologie noch Religion im Orcus von Geschichte und Tradition verschwinden, ganz im Gegenteil, entscheidend war vielmehr die Entgegensetzung von (fortschrittlichem) Wissen und (konservativem) Glauben. Nur dann, so die Argumentationslogik der ,nomenclatura accurata“, war der instrumentellen Vernunft der Platz zu sichern, der ihr gebührte und der Mensch als das zu begreifen, was er war: nämlich als homo rationalis sensibilis. ${ }^{12}$

Wegweisend oder neuartig ist vor allem sein Versuch, die rechtlichen, ökonomischen und politischen Strukturen der Moderne und deren legitimationstheoretischen Implikationen systematisch und d.h. für Hobbes strategisch zu begründen. Fragen der indivi-

8 Die Literatur zu T. Hobbes' staats- und souveränitätstheoretischer Konzeption ist inzwischen Legion; verwiesen sei an dieser Stelle nur auf E.-W. Böckenförde, Sicherheit und Selbsterhaltung vor Gerechtigkeit, Basel 2004; W. Kersting, Thomas Hobbes, Hamburg 2002; K.-M. Kodalle, Wahrheit und System, Köln 1972; C. Schmitt, Der Leviathan, Hamburg 1938; Q. Skinner, Freiheit und Pflicht, Frankfurt a.M. 2008 sowie B. Willms, Thomas Hobbes. Das Reich des Leviathan, München 1987; zum Urbild des modernen Staates und seinen Gegenbildern aus der Perspektive einer politischen Ikonographie H. Bredekamp, Thomas Hobbes, Leviathan, Berlin 2006.

9 Grundlegend dazu immer noch M. Foucault, Die Ordnung der Dinge, Frankfurt a.M. 1995, S. $46 \mathrm{ff}$. und öfter.

$10 \mathrm{Zu}$ Genese, Struktur und Auflösung dieses Funktionszusammenhanges vgl. etwa E. Cassirer, Individuum und Kosmos, Leipzig 1927, S. 7 und öfter sowie E. Friedell, Kulturgeschichte der Neuzeit, München 1931, S. 173 ff.

$11 \mathrm{Zu}$ den Standards und Methoden der damaligen Wissenschaftskonzepte und -transfers siehe H. Blumenberg, Die Legitimität der Neuzeit, 1988, S. 20 ff. und öfter sowie ders., Die Genesis der kopernikanischen Welt, Frankfurt a.M. 1975, S. 147 ff. und öfter; zu Hobbes' Systemsund Prinzipienideal Willms (Fn. 8), S. 61 ff.

$12 \mathrm{Zu}$ der damit einhergehenden Problematik eines selbst unreflektierten Wissenschafts- und Fortschritts-Glaubens vgl. P. Stekeler-Weithofer, Philosophie des Selbstbewusstseins, Frankfurt a.M. 2005, S. 270 ff. und öfter; zum Verhältnis von Empirismus und Rationalismus aus philosophiegeschichtlicher Perspektive K. J. Engfer, Empirismus vs. Rationalismus?, Paderborn 1996, S. 11. 
duellen Sinnorientierung, aber auch der gesellschaftlichen Sozialkontrolle sind für ihn nicht länger auf ein metaphysisches Ideal der menschlichen Natur zu beziehen, sondern an den rationalisierten Bedürfnissen der einzelnen Akteure auszurichten. Demgemäß ist für Hobbes praktische Philosophie, im Sinne einer Theorie des Politischen, immer auch analytische Friedenswissenschaft; und die Methode des mos geometricus die heuristische Grundlage, mittels derer die normativen Ansprüche einer monopolisierten Gewalt und folglich allgemein anerkannte Rechte und Pflichten begründet werden können. In den Worten von Hobbes: „Der alleinige Weg zur Errichtung einer solchen allgemeinen Gewalt, die in der Lage ist, die Menschen vor dem Angriff Fremder und vor gegenseitigen Übergriffen zu schützen und ihnen dadurch eine solche Sicherheit zu verschaffen, dass sie sich durch eigenen Fleiß und von den Früchten der Erde ernähren und zufrieden leben können, liegt in der Übertragung ihrer gesamten Macht und Stärke auf einen Menschen oder eine Versammlung von Menschen. [...] Ist dies geschehen, so nennt man diese zu einer Person vereinigte Menge Staat [...] Dies ist die Erzeugung jenes großen Leviathan oder besser, um es ehrerbietiger auszudrücken, jenes sterblichen Gottes, dem wir unter dem unsterblichen Gott unseren Frieden und Schutz verdanken“. ${ }^{13}$

Die Zielrichtung der Argumentation ist klar: Ordnungspolitik ist notwendig Sicherheitspolitik, eine Alternative dafür gibt es nicht. Der Einzelne als atomisierter Akteur eines auf sich selbst verwiesenen Gemeinwesens begibt sich jeglicher Möglichkeiten der Risiko- und Konfliktregulierung, der Staat mit seinem Gewaltmonopol bietet umfassenden Schutz. Zur Geltung gebracht ist mit dieser Verknüpfung von Leistung und Legitimation ein staatstheoretisches Paradigma, das in seiner Grundstruktur, und gerade darin besteht die Bedeutung dieser kritischen Macht- und Vernunftkonzeption, auch heute noch Gültigkeit beanspruchen kann. Mit der kompositorischen Methode des mos geometricus verlässt Hobbes, ebenso wie Machiavelli, das zoon politikon-Theorem aristotelischer Provenienz. ${ }^{14}$ Ausschlaggebend ist nun ein am Nutzenkalkül ausgerichteter Individualismus; ein Individualismus, der in der liberalistischen Tradition eines Locke, Hume oder Mill seine Fortsetzung finden wird. Der Mensch zerfällt gewissermaßen in zwei Teile: Der erste kennzeichnet ihn als Sinnenwesen mit empfindsamer Sentimentalität und formuliert damit das Bild eines auf Ausgleich bedachten Gutmenschen. Der zweite Teil fokussiert auf ein Wesen, das auf berechnende Weise seine Vorteile maximiert. Hier wird er zum homo oeconomicus, vor dem man sich durchaus in Acht zu nehmen hat. Dennoch unterscheidet sich Hobbes' Modell deutlich von dem Machiavellis. Steht bei Hobbes die Balance von Vernunft, Macht resp. Autorität und Unterordnung im Vordergrund, so geht es Machiavelli ganz offenkundig um eine funktionale und nicht weniger aggressive „Klugheitspragmatik“, d.h. um Leitlinien einer

13 Leviathan oder Stoff, Form und Gewalt eines kirchlichen und bürgerlichen Staates, Frankfurt a.M. 1996 (1651), S. 134 ff.

14 Zum zoon politikon-Theorem in seiner aristotelischen Ausformung und mittelalterlich-christlichen Rezeption vgl. Ch. Grawe/A. Hügli, Artikel: Mensch, in: HWdPhil Bd. 5, Basel 1980, Sp. 1061 ff.; W. Gebauer, Die Aufnahme der Politik des Aristoteles und die naturrechtliche Begründung des Staates, Stuttgart 1936; O. Höffe, Praktische Philosophie, das Modell Aristoteles, Berlin 2008; W. Kullmann, Aristoteles und die moderne Wissenschaft, Stuttgart 1998 sowie J. Ritter, Metaphysik und Politik, Frankfurt a.M. 1969, S. 106 ff. 
politischen Selbstbehauptungs- und Machtsteigerungslehre. ${ }^{15}$ Gerade dieser völligen Entgrenzung jeglicher Vernunft- und Autoritätsdiskurse zugunsten frei flottierender Ideologeme will das hobbessche Leistungs-Legitimations-Paradigma entgegentreten und so zugleich der immanenten Logik von Konflikt und Intervention, von Gewalt und Vorbeugung einen transparenten Normkontext zur Seite stellen.

Die staatstheoretischen Debatten der Folgezeit, vor allem im Übergang von einer (rein) szientistischen zu einer personalen Wissensorganisation, können als unmittelbare Reaktion auf jene innovative Verknüpfung von Sicherheitsleistung und Macht- resp. Herrschaftslegitimation und damit ebenso als eine Strukturgeschichte der (politischen) Vernunft gelesen werden. ${ }^{16}$ In der Konsequenz wird das Rechtsgewährleistungsparadigma immer wieder modifiziert und so den Sinnpotentialen moderner Gesellschaften angepasst, man denke hier nur an die Legitimationserwägungen bei Locke, Pufendorf, Montesquieu oder Rousseau. ${ }^{17}$ An zentrale Stelle rückt nun ein am Immanenz- und Säkularisierungsgedanken orientierter Interessenausgleich zwischen dem Herrschaftsund Disziplinierungsanspruch des Staates und dem Autonomie- und Emanzipationsbegehren des Einzelnen. Waren die „großen Erzählungen“ (Lyotard) mit ihren naturrechtlich orientierten Sinnstiftungsfunktionen schon bei Hobbes weitgehend marginalisiert, so treten sie jetzt noch stärker in den Hintergrund. ${ }^{18}$ Im Gegenzug kristallisiert sich ein vernunftkritisches Verständnis von Moral und Recht heraus. Letzteres führt zu einer Personalisierung und auf diesem Wege zu einer kategorialen Neubestimmung des Leistungs-Legitimations-Paradigmas, ein (Selbst-)Verständnis, das sich auch in einer entsprechenden Daseins- und Konfliktanalyse niederschlagen muss. Gesucht wird nunmehr eine Form des Zusammenschlusses, „die“, so Rousseau, „mit ihrer ganzen gemeinsamen Kraft die Person und das Vermögen jedes Einzelnen verteidigt und schützt und durch die doch jeder, indem er sich mit allen vereinigt, nur sich selbst gehorcht und

$15 \mathrm{Zu}$ Machiavellis Konzept einer funktionalen Macht- bzw. Wertebegründung und deren aktuelle Bedeutung siehe I. Berlin, Das krumme Holz der Humanität, Frankfurt a.M. 1992, S. 21 ff.; H. Münkler, Machiavelli, Frankfurt a.M. 2004; D. Sternberger, Drei Wurzeln der Politik, Frankfurt a.M. 1984 sowie J. Taubes, Religionstheorie und politische Theologie, München 1983.

16 Als eine solche Konzeption des Übergangs muss die ,Szienza nova' Giambattista Vicos von 1725 angesehen werden. Sichtbar wird in der ,Szienza nova' bereits die Loslösung von einer allzu szientistisch-formalen und die Hinwendung zu einer auch kulturgeschichtlichen Begründung menschlicher Wissens- und Lebensformen. Gleichzeitig bleibt eine Vielzahl metaphysischer Grundannahmen erhalten, die vor allem den personal-freiheitlichen Aspekt moderner Praxis- und nicht zuletzt Rechts(verfahrens)organisation verdunkeln. $\mathrm{Zu}$ den hier durchaus interessanten Transformationslinien siehe M. Gans, Das Subjekt der Geschichte, 1993, S. 19 und öfter.

17 Die Entwicklung dieser staats- und legitimationstheoretischen Konzepte zeichnet $R$. Zippelius in seiner Analyse anschaulich nach, siehe Geschichte der Staatsideen, München 2003, dort insbes. S. $97 \mathrm{ff}$.

18 Das postmoderne Wissen, 1986, S. 14. J.-F. Lyotards Analyse widmet sich zwar vornehmlich der Wissensorganisation spät- bzw. postmoderner Lebensformen, die aufgezeigten Entwicklungslinien markieren aber die auch für den vorliegenden Zusammenhang wichtigen Legitimationsdiskurse und die damit verbundenen Transformationsstrategien. Zum kultur- und mentalitätsgeschichtlichen Hintergrund vgl. J. Huizinga, Herbst des Mittelalters, Stuttgart 2006; die aktuelle Debatte beleuchtet R. Pippin, Die Verwirklichung der Freiheit, Frankfurt a.M. 2005, S. 191 ff. und Taylor (Fn. 1). 
genauso frei bleibt wie zuvor."19 Und Rousseau findet diese Form im Gesellschaftsvertrag und dem damit verbundenen Gemeinwillen. Denn damit der Gesellschaftsvertrag keine Lehrformel bleibt, ,schließt er stillschweigend eine Übereinkunft ein, die allein die anderen ermächtigt, dass, wer sich weigert dem Gemeinwillen zu folgen, von der gesamten Körperschaft dazu gezwungen wird“. ${ }^{20}$

Die kategoriale Neubestimmung der Gewährleistungsstrategien ist als Paradigmenwechsel innerhalb des Paradigmas zu verstehen. Die Matrix von Leistung und Legitimation wird hier unmittelbar an die je individuellen Selbstbestimmungspraxen des Menschen geknüpft. Verabschiedet wird damit nicht nur die Bürgerkriegsmetaphorik hobbesianischer Provenienz, sondern auch die zugrunde gelegte Anthropologie des homo homini lupus. ${ }^{21}$ Das Freiheitsargument tritt so aus dem Schatten paternalistisch, vor allem aber autoritär organisierter Verhaltenskontexte und -codices und schafft insoweit die Basis für ein neues Selbst-Bild des Akteurs als handelndes Subjekt und Teilnehmer an einer gemeinschaftlichen Kultur des Redens und Urteilens. ${ }^{22}$ Personale Autonomisierungsstrukturen werden dadurch zum Ausgangs- resp. Fluchtpunkt einer Identitäts- und Statusbegründung und formulieren gerade damit ein neues Staats- und Gesellschaftsverständnis. ${ }^{23}$ Denn, so die Formulierung Kants „Freiheit ist [...] sofern sie mit jedes anderen Freiheit nach einem allgemeinen Gesetze zusammen bestehen kann, dieses einzige, ursprüngliche, jedem Menschen, kraft seiner Menschheit zustehende

19 Vom Gesellschaftsvertrag oder Grundsätze des Staatsrechts (Contrat social), 1994 (1762), S. 17.

20 A.a.O., S. 21.

21 Speziell zur Anthropologie von Thomas Hobbes W. Bartuschat, in: Höffe (Hrsg.), Thomas Hobbes, Anthropologie und Staatsphilosophie, Freiburg 1981, S. 19 ff. Das Verhältnis staatsund politiktheoretischer Begründungsformen zu ihren anthropologischen Implikationen analysieren Berlin (Fn. 15), S. 37 ff. und öfter; W. Brugger, Das anthropologische Kreuz der Entscheidung in Politik und Recht, Baden-Baden 2008; D. Jörke/B. Ladwig, Politische Anthropologie, Baden-Baden 2009 und H. Ryffel, Grundprobleme der Rechts- und Staatsphilosophie. Philosophische Anthropologie des Politischen, Neuwied 1969, S. $101 \mathrm{ff}$.

22 Zur Genese des Autonomie- und Freiheitsarguments als fundamentale Kategorie moderner Subjekt- und Praxisphilosophie P. Bieri, Das Handwerk der Freiheit, München 2001; V. Gerhardt, Selbstbestimmung, Stuttgart 2001; D. Henrich, Konstellationen, Stuttgart 1991; ders., Selbstverhältnisse, Stuttgart 2001; J. McDowell, Geist und Welt, Paderborn 1998; Pippin (Fn. 18), S. 71 ff.; M. Seel, Sich bestimmen lassen, Frankfurt a.M. 2002, S. 279 ff.; StekelerWeithofer (Fn. 12), S. 337 ff. und öfter sowie C. Taylor, Quellen des Selbst, Frankfurt a.M. 1994; aus sprachanalytischer Perspektive argumentieren vor allem E. Tugendhat, Selbstbewusstsein und Selbstbestimmung, Frankfurt a.M. 1979 und L. Wittgenstein, Philosophische Untersuchungen, in: ders., Werkausgabe, Bd. 1, Frankfurt a.M. 1984.

23 Die Entwicklung und Bedeutung freiheitsphilosophischer Rechtskonzepte untersucht $K$. Kühl, Freiheitliche Rechtsphilosophie, Baden-Baden 2008; insbesondere zu Kant siehe $W$. Kersting, Wohlgeordnete Freiheit. Immanuel Kants Rechts- und Staatsphilosophie, Paderborn 2007. 
Recht." 24 Wechselseitige und institutionalisierte Rechte und Pflichten fungieren deshalb, entgegen Hobbes, nicht allein als Oktroi und d.h. als subjektgelöste Normbefehle, sondern vor allem als frei gewählte Orientierungen verobjektivierter Anerkennungsspielräume. ${ }^{25}$

Angesprochen ist dabei ein (transzendentales) Modell des Rechts, das, trotz seiner notwendig formalen und verfahrensgeleiteten Struktur, immer auch an die performativ geäußerte, in der Regel gelebte Akzeptanz der Menschen gebunden und in diesem Sinne praktisch gewordene Vernunft ist - oder jedenfalls sein soll. Zugleich, und darin liegt die Kernaussage dieses Konzepts, können die Akteure als rechtlich identifizierbare Urheber ihres Handelns und Unterlassens, ihres Wissens oder Unwissens bestimmt werden. ${ }^{26}$ So gelingt es auch, Konflikte oder Verletzungshandlungen auf praxis- und wertbezogene Gründe zurückzuführen, Gründe, die es, je nach Ausgestaltung des positiven (Straf-)Rechts, ermöglichen, Qualität und Maß des Schadens resp. des Unrechts zu ermitteln. Konflikte beruhen also auf Freiheitsanmaßungen, die in der Regel mit Anerkennungsmissachtungen einhergehen. Deshalb verläuft auch die Risiko- und Konfliktanalyse „Zweistufig“. Zum einen geht es um die verfahrensgeleitete Offenlegung und entsprechende Restitution der Freiheitsverletzung, zum anderen um Fragen der Erwartungssicherung und Normstabilisierung. Letzteres macht vor allem deutlich, dass damit die Relation von Konflikt, Unrecht und Prävention, von Gefahr, Gewalt und Vorbeugung, durch das Kriterium der personalen Urheberschaft konkretisiert und schließ-

24 Metaphysik der Sitten, GS, Berlin 1902 ff., ND 1977 (1797), AA VI, S. 237. Kurz zuvor hatte Kant die analytische Verknüpfung von Rechtsgesetzen der Freiheit und ihrem Zwangsmoment hervorgehoben: „Das Recht ist mit der Befugnis zu zwingen verbunden. Der Widerstand“, so Kant, ,,der dem Hindernisse einer Wirkung entgegengesetzt wird, ist eine Beförderung dieser Wirkung und stimmt mit dieser zusammen. Nun ist alles, was unrecht ist, ein Hindernis der Freiheit nach allgemeinen Gesetzen. Der Zwang aber ist ein Hindernis, der der Freiheit geschieht. Folglich: wenn ein gewisser Gebrauch der Freiheit selbst ein Hindernis der Freiheit nach allgemeinen Gesetzen (d.i. unrecht) ist, so ist der Zwang, der diesem entgegengesetzt wird, als Verhinderung eines Hindernisses der Freiheit mit der Freiheit nach allgemeinen Gesetzen zusammen stimmend, d.i. recht [...].“ AA VI, S. 231.

25 Zum Begriff der Orientierung als philosophische und rechtliche Kategorie der Interessens-, Wissens- und Handlungskoordination W. Stegmaier, Philosophie der Orientierung, Berlin, New York 2008, dort insbesondere S. 460 ff.; zum philosophischen Modell der Anerkennung B. Merker (Hrsg.), Subjektivität und Anerkennung, Paderborn 2004, A. Honneth, Kampf um Anerkennung, Frankfurt a.M. 1992 sowie $P$. Ricoer, Wege der Anerkennung, Frankfurt a.M. 2006; zur Bedeutung resp. Verarbeitung dieses Konzepts im Recht und angrenzenden Wissenschaften W. Schild (Hrsg.), Anerkennung, Würzburg 2000.

26 Eine Rekonstruktion des Urheberbegriffs in freiheitsphilosophischer Lesart unternimmt Gerhardt (Fn. 22), S. 187 ff. und öfter. 
lich auf das normative Potential der jeweils gelebten oder als gelebt unterstellten Ordnung bezogen wird. ${ }^{27}$

Aus der Sicht des 20. und 21. Jahrhunderts, oder anders gesagt, aus Sicht einer sich selbst problematisch gewordenen Vernunft- und Fortschrittsgeschichte, kann dieses Modell auch als klassisch aufgeklärter Idealtypus moderner Rechtsgewährleistung und damit zugleich als Bezugs- oder, je nach Perspektive, als Fluchtpunkt aller weiteren Argumentationsmuster verstanden werden. Insofern ist, jedenfalls ideengeschichtlich und staatstheoretisch, keineswegs entscheidend, ob das Modell in den Gesellschaften, die häufig mit der Epoche der europäischen Aufklärung oder des Deutschen Idealismus identifiziert werden, annähernd oder gar vollständig realisiert war. Ausschlaggebend ist vielmehr, inwieweit damit Prinzipien des Redens und Urteilens, des Verstehens und Handelns formuliert wurden, die, trotz des zugestandenen Anspruchs der vorgetragenen Wissensanalyse, immer auch als wissenschaftlich kontrovers oder als realpolitisch uneinlösbar begriffen wurden. ${ }^{28}$ In welcher Form solche Gegenläufigkeiten auf das Selbstverständnis von Recht und Staat durchschlagen können, zeigt sich nicht zuletzt an einer Neujustierung, die das freiheitsphilosophische Design des Leistungs-Legitimations-Paradigmas und die entsprechende Konfliktverarbeitung im Verlaufe des 19. Jahrhunderts erfuhr; eine Entwicklung, die in ihrer Bedeutung gar nicht überschätzt werden kann.$^{29}$

Galt nämlich der Rechtsstaat, gerade in der Semantik der Aufklärung, als Repräsentation des Allgemeinwillens, ${ }^{30}$ als, um mit den Worten Hegels zu sprechen, „Dasein der Freiheit" 31 so wird er, nicht nur in den Augen professioneller Juristen oder diverser politischer Eliten, zunehmend als Organisations- und Verwaltungsstaat wahrgenommen. Wie viele Entwicklungen im öffentlichen Raum des Rechts, so beginnen aber auch hier die Weichenstellungen weit vor ihrer gesellschaftlichen Verortung und politischen Institutionalisierung. Entsprechende Ansätze werden, soweit man sich genauer umschaut, bereits bei Beccaria, Grolman oder Feuerbach für das Strafrecht ${ }^{32}$ oder bei

$27 \mathrm{Zu}$ den Zurechnungs- und Verantwortungs(zuweisungs)strategien freiheitsphilosophischer Schuld-, Straf-bzw. Sanktionsmodelle etwa $K$. Günther, Schuld und kommunikative Freiheit, Frankfurt a.M. 2005; G. Jakobs, Norm, Person, Gesellschaft, Berlin 2008; S. 80 ff.; M. Kahlo, Die Handlungsform der Unterlassung als Kriminaldelikt, Frankfurt a.M. 2001, S. 181 ff.; M. Köhler, Der Begriff der Strafe, Heidelberg 1986; W. Lübbe, Verantwortung in komplexen kulturellen Prozessen, Freiburg 1998; M. Pawlik, Person, Subjekt, Bürger, Berlin 2004; W. Schild, Der Strafrichter in der Hauptverhandlung, Heidelberg 1983, S. 7; K. Seelmann, Anerkennungsverlust und Selbstsubsumtion, Freiburg 1995, S. 11 ff.; S. Stübinger, Das „idealisierte“ Strafrecht, Frankfurt a.M. 2007, S. 288 ff. und öfter und Verf., Schuldtypisierung als Begriffsanalyse, Berlin 2007, S. $32 \mathrm{ff}$.

28 Vor allem gegenüber den Modellen von Kant, Fichte und Hegel wurde dieser Einwand immer wieder geführt. Zu dieser Debatte und ihren Konsequenzen H.-D. Klein (Hrsg.), Systeme im Denken der Gegenwart, Bonn 1993 und Stekeler-Weithofer (Fn. 12), S. 15 ff.

29 Zur Entwicklung der (Straf-)Rechtswissenschaften und dem damit einhergehenden Selbstverständnis als „Anwendungswissenschaft“ siehe Verf. (Fn. 27), S. 68, 98 ff.

30 Vgl. dazu die Darstellung der rousseauschen Position im Text.

31 Grundlinien der Philosophie des Rechts, Frankfurt a.M. 1970 (1821), § 29.

32 Vgl. C. Beccaria, Von den Verbrechen und von den Strafen, 1764, ND Berlin 2005; K.-L. v. Grolman, Grundsätze der Criminalwissenschaft, 1798, ND Goldbach 1998 und P.J.A. v. Feuerbach, Revision der Grundsätze und Grundbegriffe des positiven peinlichen Rechts, 1799/ 1800, ND Aalen 1970. 
Sonnenfels, Justi und Hufeland für die Staatswissenschaften diskutiert. ${ }^{33}$ Besonders deutlich wird der Strukturwandel schließlich bei v. Mohls Polizei-Wissenschaft und Jellineks Staatslehre. ${ }^{34}$ Juristische Argumentations- und ordnungspolitische Erklärungsmuster lösen sich nunmehr von ihren philosophischen Begründungskontexten. ${ }^{35}$ Die moderne Jurisprudenz entwickelt sich zur praktischen Orientierungs- und instrumentellen Anwendungswissenschaft. Sie vertraut dem esprit positif(Comte), den Möglichkeiten einzelwissenschaftlicher Welterkenntnis und wird in diesem Sinne funktional. ${ }^{36} \mathrm{Im}$ Mittelpunkt steht nicht mehr die vernunftgenormte Erfassung sinnorientierter Rechts- und Lebensformen, sondern die Ausweitung gesellschaftsstabilisierender Interventions- und Steuerungspraktiken. Im Stile einer Funktionseinheit firmiert der Staat, oder das, was man nun als Staat definiert, selbst als handelnder Akteur und wird so als Agent eigens formulierter Interessen behauptet. „Aus dem Rechtsstaat, dem Nichts-alsRechtsstaat“, so v. Liszt, „der sich damit begnügt hat oder wenigstens damit begnügen wollte, dem freien Spiel der Kräfte seinen ruhigen Lauf zu sichern [...], hat sich im Laufe der Jahrzehnte der moderne Verwaltungsstaat entwickelt, der durch bewusste Zwecksetzung eingreift in das freie Spiel der Kräfte, eingreift, um auf der anderen Seite die Interessen der Gesamtheit gegenüber dem Übermut des Einzelnen wahrzunehmen." ${ }^{37}$ Das bedeutet jedoch nicht, dass die Stabilisierung von Normen und die SelbstVersicherung von Gesellschaften allein auf horizontale Interventions- und Herrschaftsstrategien beschränkt wäre, im Gegenteil, ergänzt werden diese Strategien durch ein

33 Siehe $J$. v. Sonnenfels, Grundsätze der Polizei, Bd. 1, 1787, ND München 2003, J. H. G. v. Justi, Die Grundfeste zu der Macht und Glückseligkeit der Staaten, 1760, ND Aalen 1965 und G. Hufeland, Lehrsätze des Naturrechts und der damit verbundenen Wissenschaften, 1790, ND Glashütten i.T. 1973.

34 Die Titel lauten vollständig: Die Polizey-Wissenschaft nach den Grundsätzen des Rechtsstaates, Tübingen 1832/33 und Allgemeine Staatslehre, Berlin 1914.

35 Freilich gibt es Ausnahmen: Zu ihnen zählen H. F. K. v. Stein und W. v. Humboldt, mit Einschränkungen wohl auch P.J.A. v. Feuerbach. Vor allem bei Humboldt und seinen „Ideen zu einem Versuch, die Grenzen der Wirksamkeit des Staates zu bestimmen“von 1792 (Werke Bd. 1, 2002, S. 56 ff.) kann man erkennen, wie politischer Pragmatismus und freiheitliche Selbstorganisation des Menschen miteinander vermittelt und so zugleich gegen einen alles regulierenden (Obrigkeits-)Staat gewendet werden können.

36 „Voir pour savoir, savoir pour prevoir, prevoir pour prevenir“, heißt es dann in der Soziologie A. Comtes, Stuttgart 1974 (1842), S. 470. Die Entwicklung von philosophischem Positivismus und moderner Einzelwissenschaft untersuchen B. Plé, Die „Welt“ aus den Wissenschaften, Stuttgart 1996 und G. Winkler, Rechtstheorie und Erkenntnislehre, Wien 1990, dort insbesondere auf die Reine Rechtslehre H. Kelsens bezogen.

37 Mitgeteilt durch die IKV, Bd. 19, 1912, S. 377. Interessant ist freilich, dass sich, parallel zu dieser rechts- und ordnungspolitischen „Bestandaufnahme“, vor allem soziologische Konzepte darum bemühen, das Verhältnis von Gemeinschaft und Gesellschaft neu zu bestimmen. Vor dem Hintergrund sich wandelnder ökonomischer und sozialer Strukturen geht es nunmehr darum, der „Entmaterialisierungstendenz“ des modernen Staates, also dem, was v. Liszt mit dem Verwaltungsstaat bezeichnet, etwas entgegenzusetzen oder, um es mit einem Wort $\mathrm{He}$ gels zu sagen, nach der Sittlichkeit der modernen Lebens- und Kooperationsverhältnisse zu suchen. Prominent sind hier immer noch die Analysen von F. Tönnies, Gemeinschaft und Gesellschaft, Leipzig 1887, E. Dürkheim, Erziehung, Moral, Gesellschaft, Neuwied 1973 (1902/ 03) und von G. Simmel, Soziologie. Untersuchungen über die Formen der Vergesellschaftung, Leipzig 1908; zum Ganzen jetzt auch H. Rosa et al., Theorien der Gemeinschaft, Frankfurt a.M. 2010, passim. 
neues Subjekt- und Personenverständnis. Letzteres konstruiert den Akteur gerade auch als atomisiertes Selbst-Organisations- und Kontrollsystem, das in den (ökonomisierten) Kontexten des je eigenen Lebens spezifische Kompetenzen und wissens- oder autoritätsbasierte Positionen einsetzt - und konsolidiert so das komplexe Ordnungsgefüge auf vertikaler Ebene. $^{38}$

\section{Orientierungsmuster der Gegenwart}

Zur Geltung gebracht ist damit eine Norm-, Person- und Gesellschaftslegitimation, die, wie man mit Foucault sagen kann, als Wechselwirkung zwischen zwei Orientierungsmustern, zwischen Macht- oder Herrschaftstechniken einerseits und Selbsttechniken andererseits, beschrieben werden muss. ${ }^{39}$ „Der Kontrapunkt“, so Foucault, ,an dem die Form der Lenkung der Individuen durch andere mit der Wiese der Selbstführung verknüpft ist, kann [...] Regierung genannt werden. In der weiten Bedeutung des Wortes ist Regierung nicht eine Weise, Menschen zu zwingen, das zu tun, was der Regierende will; vielmehr ist sie immer ein bewegliches Gleichgewicht mit Ergänzungen und Konflikten zwischen Techniken, die Zwang sicherstellen und Prozessen, durch die das Selbst durch sie selbst konstruiert oder modifiziert wird." 40

Genau besehen handelt es sich hier um eine Logik der Macht- und Normlegitimation, die sich, wenngleich in veränderter Gestalt, auch in den (ökonomischen) Strukturen des Rechts-, Sozial- und Versicherungsstaates des 20. bzw. 21. Jahrhunderts fortgeschrieben hat. Denn auch dieses Ordnungskonzept agiert weitgehend funktional, nicht zuletzt um die permanent anfallenden „Produktionskosten“ individueller wie kollektiver Freiheitsspielräume bestimmen und realisieren zu können. ${ }^{41}$ Diese (Re-) Strukturierung des ordnungspolitischen Terrains ist auch staatstheoretisch nicht uninteressant: Denn an der Oberfläche oder, wenn man will, idealtypisch, orientiert sich der moderne Rechts- und Sozialstaat nach wie vor an der „klassisch aufgeklärten“ Staats(zweck)theorie. ${ }^{42}$ Be-

38 Analysiert wird diese Entwicklung von T. Lemke, Eine Kritik der politischen Vernunft, Berlin usw. 1997, S. 126 ff. und öfter.

39 Vgl. hier nur: Der Wille zum Wissen, Frankfurt a.M. 1977, S. 121 ff.; ders., Der Gebrauch der Lüste, Frankfurt a. M. 1986, 315 ff. und ders., Freiheit und Selbstsorge, Frankfurt a.M. 1993, S. $32 \mathrm{ff}$.

40 Political Theory, Vol. 21 (1993), S. 198, 203.

41 Dezidiert schon die Analyse bei $M$. Weber: „Es kommt also zunächst wieder darauf an: die besondere Eigenart des okzidentalen und, innerhalb dieses, des modernen okzidentalen, Rationalismus zu erkennen und in ihrer Entstehung zu erklären. Jeder solche Erklärungsanspruch muss, der fundamentalen Bedeutung der Wirtschaft entsprechend, vor allem die ökonomischen Bedingungen berücksichtigen. Aber es darf auch der umgekehrte Kausalzusammenhang darüber nicht unbeachtet bleiben. Denn wie von rationaler Technik und rationalem Recht, so ist der ökonomische Rationalismus auch von der Fähigkeit und Disposition der Menschen zu bestimmten Arten praktisch-rationaler Lebensführung überhaupt abhängig. Gesammelte Aufsätze zur Religionssoziologie I, Tübingen 1920, S. 12; instruktiv dazu auch $M$. Stolleis, Konstitution und Intervention, Frankfurt a.M. 2001, passim.

$42 \mathrm{Zu}$ Genese und Selbstverständnis der Staats(zweck)lehre E.-W. Böckenförde, in: Ehmke (Hrsg.), Festschrift für Arndt, Frankfurt a.M. 1969, S. 53 ff.; M. Friedrich, Geschichte der deutschen Staatsrechtswissenschaft, Berlin 1997 und F. Hespe, Zur Entwicklung der Staatszwecklehre in der deutschen Staatsrechtswissenschaft, Göttingen 1964. 
sonders deutlich wird das an der Formulierung des Grundrechtskatalogs der Art. $1 \mathrm{ff}$. GG etc. Die Tiefenstrukturen, d.h. die Pragmatik gesellschaftlicher Funktionszusammenhänge, verweisen allerdings auf eine andere, zumindest parallel mitgeführte Semantik und insofern auf die heute üblichen Interventions- und Steuerungspraktiken, man erinnere sich nur an die Rede von der so genannten „Ich-AG“, von notwendigen „Überwachungs- und Visualisierungstechniken“ des Alltags oder diverse „biopolitischen Strategien" des gouvernementalen Managements. Welche Probleme das erzeugen kann oder welche Spannungen das impliziert, zeigt, jedenfalls verfassungsdogmatisch, der aktuelle Streit um eine zeitgemäße und interessensangemessene Interpretation von Art. 1 Abs. 1 GG. ${ }^{43}$ Nicht weniger brisant sind die immer wieder auftretenden Friktionen und Gegenläufigkeiten in der gegenwärtigen Gesundheits-, Kriminal- oder Sozial(fürsorge)politik. ${ }^{44}$ Offenkundig wird so der Wandel des Leistungs-Legitimations-Paradigmas: von der ordnungspolitischen Neuvermessung bei Hobbes, der freiheitstheoretischen Reformulierung bei Rousseau, Kant und Hegel, bis hin zur Ausgestaltung im liberalen Staat des 19., vor allem aber des 20. und 21. Jahrhunderts. Hintergrund dieser Transformationen ist ein verändertes Konflikt- und Risikoverständnis.

\section{Zur Struktur der modernen Risiko- und Interventionsstrategien}

\section{Die Umcodierung des traditionellen Sicherheitsdispositivs im modernen Interventionsstaat}

Das moderne Risiko- und Interventionsverständnis beruht auf der Umcodierung des traditionellen Sicherheitsdispositivs. ${ }^{45}$ Während die „klassisch aufgeklärte“ Rechts- und Staatstheorie und das damit einhergehende Sanktionenrecht die Verarbeitung von Risiken und Konflikten an einen starken Personen-, Freiheits- und Vernunftbegriff knüpfte und auf diese Weise die Areale von Innen und Außen, von Moral und Recht und schließlich von Repression und Prävention - Polizei- und Strafrecht - unterschied, glaubt der moderne Sozial- und Verwaltungsstaat, Sicherheit und gesellschaftliche Sinnproduktion nur gewährleisten zu können, indem er die Techniken der Interventi-

43 Vgl. dazu die Kommentierung von M. Herdegen in: Maunz/ Dürig, 44. Lfg. 2005, Art. 1 Abs. 1 GG (vgl. aber bereits die 42. Ergänzungslieferung von 2003) und die Reaktion von $E$.-W. Böckenförde in der FAZ vom 3. September 2003, ausgearbeitet in: Blätter für deutsche und internationale Politik, 2004, S. 1216 ff.; abrufbar unter: www.wissensgesellschaft.org/themen/biopolitik/menschenwürde.pdf.; der Streit- und Diskussionsstand wird zusammengefasst von C. Enders, in: Stern/ Becker (Hrsg.), Grundrechte-Kommentar, Köln 2010, Art. 1, S. 81 ff.

44 Vgl. U. Beck/ B. Bon $\beta$, Die Modernisierung der Moderne, Frankfurt a.M. 2001; P. Bourdieu, Gegenfeuer, Konstanz 2004; R. Castel, Die Metamorphosen der sozialen Frage, Konstanz 2000; Ewald (Fn. 3), S. 65 ff. und öfter; S. Krasmann/M. Volkmer (Hrsg.), Michael Foucaults ,Geschichte der Gouvernementalität' in den Sozialwissenschaften, Bielefeld 2007; S. Krasmann, Die Kriminalität der Gesellschaft, Konstanz 2003 und T. Lemke, Gouvernementalität und Biopolitik, Wiesbaden 2008, S. 89 ff. und öfter.

45 Zum Begriff des Dispositivs im Allgemeinen G. Agamben, Was ist ein Dispositiv?, Zürich 2008, S. 7; zur Verwendung bei Foucault ders., Dits et Ecrits. Schriften in 4 Bänden, Bd. 3, Frankfurt a.M. 2003, S. 392; konkret zum Titel des Sicherheitsdispositivs Lemke (Fn. 38), S. 183. 
on, d.h. den Präventions- und Vorbeugegedanken als zentrale und universell einsetzbare Normstabilisierungsmatrix zur Geltung bringt. ${ }^{46}$ Garland spricht inzwischen sogar von einer allgemeinen „Kultur der Kontrolle“. ${ }^{47}$ Dementsprechend findet sich der Präventions- und Vorbeugegedanke in den unterschiedlichsten Ressorts modern organisierter Gesellschaften. ${ }^{48}$ So wird er in der Gesundheitsförderung eng an das Modell der Früherkennung gekoppelt. Angestrebt wird mit dieser Strategie beispielsweise, „die Rate von psychischen Störungen in einem Bevölkerungssegment über einen bestimmten Zeitraum hinweg zu reduzieren, indem sie schädlichen Umständen entgegenwirkt, bevor diese eine Chance haben, Krankheiten zu produzieren. Primärprävention strebt nicht an, eine spezifische Person vor dem Krankwerden zu beschützen. Stattdessen will sie das Krankheitsrisiko für eine ganze Bevölkerungsgruppe reduzieren, so dass, obwohl einige trotzdem krank werden mögen, die Zahl der Krankheitsfälle reduziert wird. "49 Hassemer beschreibt dieses Programm für die Verbrechensprävention. Unter dem prononcierten Titel: „Sicherheit durch Strafrecht“ will er darauf hinweisen, dass das Präventionsmodell in unserem Strafrecht nicht nur faktisch mächtig, sondern, bei allen hiermit verbundenen Problemen, auch normativ begründet sei. „Es ordnet das Strafrecht einem System der Herstellung und Bewahrung von Sicherheit ein und macht es damit zu einem Instrument der Bekämpfung von Problemen und der Beherrschung von Risiken. "50 Insoweit, so Hassemer weiter, stellt es ,einen Stützpfeiler dar, den wir nicht nur einbauen müssen in jegliche Überlegung zur Zukunft des Strafrechts, sondern den wir auch begrüßen dürfen als Ausdruck einer zeitgerechten wie auch verfassungsgemäßen Strafrechtsordnung. Zu diesem Strafrecht können jedenfalls meine Augen eine Alternative nicht absehen. " ${ }^{\text {"51 }}$ Der Präventions- oder Vorbeugegedanke wird so zu einem „Vollstrecker" des umfassenden Interventions- und Steuerungsanspruchs des modernen Verwaltungsstaates; auch deshalb ist die allseits attestierte Anschlussfähigkeit dieses Paradigmas nicht besonders verwunderlich, sondern geradezu folgerichtig. ${ }^{52}$

Stark gemacht wird nun das säkularisierte oder, wenn man so will, das ,entzauberte" Sicherheitsargument. Es wird hypostasiert und damit zu einem verhandelbaren Wert

46 Dazu M. Hafen, Systemische Prävention, Heidelberg 2005, 228 ff. und öfter.

47 Kultur der Kontrolle. Verbrechensbekämpfung und soziale Ordnung in der Gegenwart. Frankfurt a.M. 2008, S. 81 ff.; darüber hinaus Frankenberg, Staatstechnik, Frankfurt a.M. 2010.

48 Vgl. hierzu M. Hafen, Grundlagen der systemischen Prävention, Heidelberg 2007; Huster (Fn. 3); G. Jakobs, Schuld und Prävention, Tübingen 1976; J. Isensee, Das Grundrecht auf Sicherheit, Berlin 1983; N. Luhmann, in: ders., Soziologische Aufklärung 2, Opladen 1991, S. 9 ff.; ders., Soziologie des Risikos, Berlin 1991; Möstl, (Fn. 2); W. Stark, in: ders., Prävention als Gestaltung von Lebensräumen, Freiburg 1988, S. 11 ff.; Stoll (Fn. 2); Wahl (Fn. 2); W. Wohlers, Deliktstypen des Präventionsstrafrechts, Berlin 2000, S. 29 ff. und öfter sowie S. Wolf(Hrsg.), Prävention im Recht, Basel 2008.

49 G. Caplan, Principles of preventive psychiatry, New York 1964, S. 26 (übersetzt v. M. Hafen).

50 HRRS 2006, 130, 143.

51 A.a.O., 139.

52 Zum Verhältnis von Anschlussfähigkeit (des Präventions- und Vorbeugekonzepts) und entsprechender Interventionsstruktur Hafen (Fn. 46), S. 233 ff. und öfter. 
„an sich“. ${ }^{53}$ Grund dafür ist die „positivistische Wende“ der Rechts- und Staatswissenschaften im 19. Jahrhundert. ${ }^{54}$ Mit der Verabschiedung metaphysischer Begründungsformen nimmt der Wert den Platz ein, den zuvor Titel wie Natur, Würde oder Geist innehatten. Die Ökonomie wird zur ,universellen Leitwährung“ und dehnt sich auf alle Bereiche menschlichen Handelns und Urteilens aus. Weil aber, so Carl Schmitt, eine „kausalgesetzliche und deshalb wertfreie Wissenschaft [...] die Freiheit des Menschen und seine religiös-ethisch-juristische Verantwortlichkeit" bedrohe, versucht man mit Wertsetzungen den Sinn menschlicher Existenz zu rechtfertigen. ${ }^{55}$ Wie alle Werte, so wird auch die Sicherheit zur jederzeit einsetzbaren Münze, mit ihr lassen sich nicht nur die unterschiedlichsten Formen der Intervention rechtfertigen, sondern auch die Strategien der Intervention und Prävention bestimmen.

\section{Vom ,Vertrauen in Gott“ zur normativen Ungewissheit im Staat}

Verständlich wird diese Entwicklung dann, wenn man sich zugleich den Wandel in der politischen wie auch rechtlichen Grammatik der Sozialkontrolle vergegenwärtigt. Das „klassisch aufgeklärte“ Staats- und Rechtsverständnis ging in der Regel davon aus, dass Konflikte und Verletzungen nicht nur normativ identifizierbar, also zurechenbar, sondern auch in ihren Folgen grundsätzlich beherrschbar sind. Erwartete oder auch eingetretene Konflikte wurden so zwar als störende, aber grundsätzlich hinnehmbare Ereignisse einer - kontrafaktisch - auf ihre Stabilität vertrauenden Gesellschaft verstanden. ${ }^{56}$

Dieses Norm- bzw. Sinnstabilisierungsmuster galt - was prima facie zu irritieren scheint - auch für mittelalterlich-christliche und neuzeitliche Rationalisierungs- und Rechtsgewährleistungskonzepte. In der Regel handelte es sich hierbei um Ordnungsmodelle, die auf zum Teil hoch komplexen Naturrechtsbegründungen beruhten, man denke hier nur an Augustinus oder Thomas auf der einen sowie Grotius oder Bodin auf der anderen Seite. Der entscheidende Unterschied zu den Konzepten der europäischen Aufklärung, des Deutschen Idealismus oder auch des (Früh-)Liberalismus lag darin, dass die basalen Strukturen der implizierten Daseins- und Vertrauensformen durch die bereits erwähnten „Metaerzählungen“ (Lyotard), also der Rede von Gott, entsprechen-

53 Zur Hypostasierung von Werten und deren Funktionalisierung im Rahmen moderner Rechtsund Staatskonzepte vgl. Schmitt, in: ders., Die Tyrannei der Werte, Hamburg 1979, S. 9 ff., der sich hier auf eine These N. Hartmanns in dessen Ethik bezog (vgl. hier die 4. Aufl., Berlin 1962, dort insbesondere S. 576); zur Genealogie des Sicherheitsbegriffs und seines Gebrauchs M. Makropoulos, in: HWdPhil Bd. 9, Basel 1995, Sp. 745 ff.

54 Vgl. dazu bereits die Ausführungen im vorangegangenen Abschnitt.

55 (Fn. 53), S. 9, 31; ähnlich M. Heidegger, Holzwege, Frankfurt a.M. 1950, S. 193, 230. Simmel wiederum analysiert diese Entwicklung aus kultursoziologischer Perspektive, ders., Philosophie des Geldes, Frankfurt a.M. 2000 (1900), S. 23 ff., 357 und 591; zum Ganzen E. Jüngel, Wertlose Wahrheit, Tübingen 2003, S. $90 \mathrm{ff}$.

$56 \mathrm{Zu}$ aktuellen Debatte M. Endress, Vertrauen, Bielefeld 2002, S. 10 ff. und öfter. 
den Heils- oder Jenseitserwartungen usw., normativ und insoweit letztbegründend abgesichert waren oder wurden. ${ }^{57}$

Ausschlaggebend für dieses Verständnis war ein im weitesten Sinne liberales Gesellschaftsmodell. Letzteres bestimmte die Bedingungen unter denen Individuen ihre Freiheitsspielräume entwickeln und erhalten konnten. Freilich, und das wird nicht immer offen gelegt, barg bereits dieses Konzept Probleme. Denn eingesetzt wurde eine Freiheit - als ,positive“ oder „negative“ sei hier dahingestellt -, die immer schon fragil und bedroht war oder jedenfalls als solche behauptet wurde und damit zum prominenten Gegenstand unterschiedlichster Interventionsstrategien werden konnte. ${ }^{58}$ Inwiefern diese Interventionen sinnvoll und angemessen waren, musste dann durch Reflexion auf die je verfügbaren Wissens- und Praxisformen bestimmt werden.

Das moderne (Straf-)Rechts- und Staatsverständnis begreift die Kontexte des Sozialen grundsätzlich als Konstellationen normativer Ungewissheit. ${ }^{59}$ Letztere werden als bedrohungsrelevante Risiken oder Gefahren reformuliert, deren Verletzungs- und Schadenspotential, so jedenfalls die Behauptung, nur durch einschneidende, insoweit aber immer auch schwer zu lancierende Interventions- und Vorbeugemaßnahmen begegnet werden kann. Das Paradigma der Vorbeugung, so betont Ewald, ,ruft eine neue Ökonomie der Rechte und Pflichten auf den Plan“, zugleich tritt mit ihm der wieder entdeckte Begriff der Ungewissheit in den Vordergrund. Letzterer, so Ewald weiter, verweise gleichsam darauf, dass eine Kontrolle des Machbaren allein durch das Wissen eine Utopie sei, also immer öfter nur vermutet oder befürchtet werden könne, vor allem aber, dass sich ein modernes Sicherheitsdenken diesen Strategien anpassen müsse. ${ }^{60}$

Gerade dieser Skeptizismus verändert auch den Geltungsbereich der Wissensproduktion, die Genese von Wissen und den Status der Wissenschaft innerhalb der gesellschaftlichen Funktionszusammenhänge. Nun ist unbestritten, dass wissenschaftliche Erkenntnisse relativ auf einem bestimmten Wissensstand sind. In ihrem Ressort bringt Wissenschaft, welcher Provenienz auch immer, zwar keine letzten Gewissheiten, aber doch ein von der wissenschaftlichen Gemeinschaft anerkanntes Referenzwissen hervor. Der Präventions- und Vorbeugegedanke jedoch provoziert immer wieder zum Vorgriff auf das, was man noch nicht weiß, ermuntert zur Berücksichtigung zweifelhafter Hypothesen und bloßer Vermutungen. Ihm wird ,Wissenschaft zum Prinzip des Misstrauens. Eines doppelten Misstrauens: gegenüber der Wissenschaft selbst, und gegenüber

57 Bei Augustinus gilt das insbesondere für ,De civitate dei' von 426; für Thomas v. Aquin sei auf dessen ,summa theologica' von 1265 verwiesen. Grotius und Bodin sind beide als brillante neuzeitliche Naturrechtstheoretiker in die Geschichte eingegangen, der erste durch seine ,Les six livres de la République' von 1576, der zweite durch die völkerrechtliche Schrift ,De jure belli ac pacis' von 1625 .

$58 \mathrm{Zu}$ den unterschiedlichen Freiheitsformen. Berlin, Freiheit. Vier Versuche, Frankfurt a.M. 1995; insbesondere S. 197 ff.; zu den entsprechenden Interventionsstrategien Foucault, (Fn. 2), S. 180.

59 A. Evers/ H. Nowotny, Über den Umgang mit Unsicherheit, Frankfurt a.M. 1987, S. 59 ff.

60 Ewald (Fn. 3), S. 5, 11; zur aktuellen Diskussion vgl. auch G. Bechmann (Hrsg.), Risiko und Gesellschaft, Opladen 1993; B. Kleinfellwonder, Der Risikodiskurs, Opladen 1995, S. 107 ff. und J. Link, Versuch über Normalismus, Opladen 1998, S. 185 ff.; aus der (Straf-)Rechtswissenschaft: C. Prittwitz, Strafrecht und Risiko, Frankfurt a.M. 1993 sowie H. Schmidt-Semisch, Kriminalität als Risiko, 2002; allgemein hierzu Wittgenstein, Über Gewissheit, in: ders., Werkausgabe, Bd. 8, Frankfurt a.M. 1989. 
den zahlreichen Evidenzen, die dafür sorgen, dass die Gesten des Alltags nicht Anlass permanenter Verwunderung sind". 61

Die Normressourcen und entsprechende Kommunikationsformen moderner Gesellschaften werden so unmittelbar an ein permanentes Verdachtsmanagement und Beeinträchtigungsrisiko geknüpft, dessen etwaige Realisierung dann als Folge individuellen oder kollektiven Handelns resp. Unterlassens gedeutet wird. ${ }^{62} \mathrm{Im}$ Zentrum stehen dabei zwei verschiedene Konfliktverarbeitungsstrategien: So kann man bereits im Vorfeld das Eintreten unerwünschter Ereignisse zu verhindern suchen oder man kann Vorsorge treffen, dass im Falle ihres Eintretens der Schaden oder das Unrecht restituiert werden kann, wobei in der Regel darauf geachtet wird, dass auch in diesen Konstellationen das Verfahren nachhaltige Wirkungen für die Zukunft verspricht. ${ }^{63}$ Unter der Hand werden damit aber auch die Grenzen zwischen den verschiedenen Normstabilisierungs- und Konfliktverarbeitungsstrategien verschliffen. Ob politische, zivil-, verwaltungs- oder strafrechtliche Konfliktlagen, entscheidend sind nicht mehr der Grad oder die Struktur der Freiheitsverletzung und die damit einhergehende Abschichtungen der Eingriffsintensitäten (Sanktionen), sondern das symbolische Potential, was die jeweilige Reaktion verspricht und das notwendig scheint, um in der (medialen) Öffentlichkeit die erwünschte Wirkung zu erzielen. In diesem Sinne, so Hassemer, ist auch „das präventive sicherheitsorientierte Strafrecht nichts weiter als das zeitgerechte Strafrecht, und die Herstellung von Sicherheit durch Strafrecht ist nichts weiter als eine vernünftige Aufgabe in Zeiten, die durch Großrisiken, Zukunftsangst, Verbrechensfurcht und Kontrollbedürfnisse gekennzeichnet sind. In dieser bedrohlichen Lage darf, so gesehen, die Strafjustiz nicht zurück stehen; sie muss sich vielmehr mit ihren Mitteln an der Sicherheitspolitik beteiligen, will sie nicht ins innenpolitische Hintertreffen geraten oder gar ihre zeitgemäßen Aufgaben verfehlen. So dürften das auch die allermeisten Strafrechtler sehen und die Innenpolitiker sowieso". 64

Diese Entwicklung offenbart jedoch ein weiteres Problem moderner Sozialkontrolle. Jenseits transzendenter Legitimationsmuster, jenseits von Religionen und sonstigen Heilserwartungen, aber auch jenseits starker Vernunftkonzepte, kann sich staatliche Macht nur noch durch einen umfassend-funktionalen Schutz individueller oder kollektiver Rechte behaupten: d.h. durch Fürsorge, Versicherung und Gefahren- oder Risikominimierung. Nichts darf außer Kontrolle geraten. Entscheidend ist dann aber immer schon die Effektivität und Durchschlagskraft des gewählten Sicherheitskalküls; eine Logik, die sich in den unterschiedlichsten Bereichen gesellschaftlicher Konfliktanalyse Bahn bricht, wie gerade die aktuellen Debatten zu politischen Ausnahmezuständen (Agamben) oder Feindkonstruktionen im Recht (Jakobs) in markanter Weise zeigen. ${ }^{65}$ Unter dem Motto: „Der Feind ist die eigene Frage als Gestalt“" wird die Unabweisbarkeit des Extremen zur Methode erhoben und so, schon aus Sicherheitsgründen, jede nur

61 Ewald (Fn. 3), S. 16, 17.

62 Dazu im folgenden Abschnitt noch genauer.

$63 \mathrm{Zu}$ den unterschiedlichen Präventionsmodellen siehe nochmals Hafen (Fn. 48), S. 132, dort auch m.w.N.

64 (Fn. 50), S. 134.

65 Siehe G. Agamben, Homo sacer, Frankfurt a.M. 2002, S. 25 und öfter; Jakobs (Fn. 27). 
erreichbare (Denk-)Möglichkeit zum Prädikat der (Rechts-)Wirklichkeit umgemünzt. ${ }^{66}$ Präventions- und Vorbeugemaßnahmen entwickeln sich damit, das hat die bisherige Analyse zeigen können, nicht nur zum „Garant“ frühzeitiger Konflikt-, Schadens- und Unrechtsminimierung, sondern auch zum omnipräsenten Code, in dem komplex organisierte Gesellschaften ihre Zukunft und das heißt vor allem: ihre Sicherheitsstandards verhandeln.

\section{Paradoxien der Interventionstechniken}

\section{Das modernes Risikomanagement zwischen Gegenwart und Zukunft}

Dieses Konzept der Konfliktverarbeitung ist alles andere als unproblematisch. Wenngleich nicht übersehen werden darf, dass komplex organisierte Gesellschaften auf diverse Steuerungs- und Interventionstechniken, also auf differenzierte Risiko- und Gefahrenabwehrmechanismen angewiesen sind. ${ }^{67}$ Ihrer Struktur nach sind Präventionsund Vorbeugestrategien vor allem eines: paradoxe Unternehmungen. Denn ihre Funktion besteht gerade darin, noch nicht vorhandene, erahnte oder nur potentielle Konflikte zu verhindern. Praevenire heißt ja bekanntlich: zuvorkommen. Das zugrunde liegende Prinzip ist nichts anderes als eine „,self-destroying prophecy“ (Bröckling). Konstruiert wird eine Zukunft und mit ihr ein Konflikt- oder Bedrohungspotential, die resp. das man um jeden Preis verhindern will. Prävention versucht im Grunde, unerwünschte Zukünfte unwahrscheinlicher zu machen. ${ }^{68}$

Genau diese Struktur forciert aber auch die enormen Schwierigkeiten. So speisen sich die Sicherheitsstrategien oder -dispositive aus mehr oder weniger gut kalkulierbaren Prognosen, nämlich aus Zukunftsannahmen, die die unüberbrückbare Differenz zwischen der gegenwärtigen Zukunft, also das, was wir heute als erwünschte oder unerwünschte Zukunft unterstellen, und der zukünftigen Gegenwart, also das, worüber wir im Grunde nichts sagen können, immer einrechnen müssen, allerdings nur selten können. Denn welche Zukunft sich als Gegenwart realisieren wird, wird man erst wissen, wenn sie nicht mehr in der Zukunft liegt und insoweit Gegenwart, gegenwärtige Gegenwart, geworden ist. ${ }^{69}$ Freilich, so Luhmann, schließt ,dieser Bruch zwischen der gegenwärtigen Zukunft und den zukünftigen Gegenwarten [...] Prognosen nicht unbedingt aus. Aber deren Wert liegt dann nur noch in der Schnelligkeit, mit der sie korrigiert werden können, und darin, dass man weiß, worauf es in diesem Zusammenhang ankommt. Es gibt also nur eine ,provisorische' Voraussicht, und ihr Wert liegt nicht in der Sicherheit, die sie gewährt, sondern in der raschen und spezifischen Anpassung an eine Realität, die anders ausfällt, als man das erwartet hätte“. 70

66 Das Zitat stammt ursprünglich von T. Däubler, wurde aber später durch C. Schmitt in den rechtswissenschaftlichen Kontext überführt; dazu unter anderem N. A. Richter, Grenzen der Ordnung, Frankfurt a.M. 2005, S. 21.

67 Siehe hierzu etwa den von N. Bröckling et al. herausgg. Sammelband, Gouvernementalität der Gegenwart, Frankfurt a.M. 2000 sowie Link (Fn. 60), S. 127 und öfter.

68 Hafen (Fn. 46), S. 250.

69 N. Luhmann, Beobachtungen der Moderne, Opladen 1992, S. 129.

70 A.a.O., S. 140. 
Zukunft ist kontingent. Dabei ist die Stoßrichtung der Prävention grundsätzlich negativ, denn sie will immer verhindern und gerade nicht schaffen. Die Zukunft, die sie antizipiert, erscheint stets finsterer als die Gegenwart, der Verzicht auf Prävention furcht- und angsterregender als deren mögliche Effekte. Auch deshalb stellt die so apostrophierte Sicherheit keinen Zustand, sondern eine nur post hoc verifizierbare Konfliktund Risikoprognose dar. Gleichwohl ist Prävention konservativ, selbst wenn sie das Leben von einzelnen Personen oder sogar Gruppen radikal verändert. In dem ihren Interventionen notwendig das prognostizierte Wissen, die Werturteile und die Machtkonstellationen der Gegenwart zugrunde liegen, projiziert sie die Gegenwart normativ auf die Zukunft. Beschrieben wird insofern nicht die (erlebte oder erlebbare) Wirklichkeit, als vielmehr eine eigens strukturierte Bedrohungswahrnehmung, die selbst erst eine „prognosekompatible Wirklichkeit" und damit die notwendige Legitimationsbasis erzeugt. Gerade aber das kann oder muss sogar, entgegen aller „Rationalisierungs- und Entzauberungsstrategien“ (Weber), zur Mystifizierung von tatsächlichen Bedrohungslagen und den entsprechenden Akteuren führen, wie sich vor allem an der bereits erwähnten „Ausnahmezustands- und Feindrechtsdebatte“ zeigen lässt. ${ }^{71}$

\section{Das anthropologische Fundament der staatlichen Vorbeugepraktiken}

Verstärkt wird die Präventionsmatrix durch das Modell einer negativen Anthropologie: In diesem Sinne liegt den Vorbeugepraktiken sowohl das Bild eines gefährdeten als auch das eines potentiell selbst gefährlichen Akteurs zugrunde. Dagegen mutet die kantische Konzeption schon fast romantisch an. Denn ihrem Fazit: „Aus so krummen Holze, als woraus der Mensch gemacht ist, kann nichts ganz Gerades gezimmert werden“ - korrespondierte immerhin das Vertrauen, dass der Mensch sich als dieses krumme Holz begreifen und seine Stellung in der Welt reflektieren kann. ${ }^{72}$ Sie unterscheidet sich aber auch von anderen, modernen Anthropologien. Hatte Gehlen den Mangel in der ,morphologischen Unspezialisiertheit" gesehen und deren Kompensation durch eine rationale und kulturimprägnierte Weltorganisation des Einzelnen behauptet, ${ }^{73}$ Plessner wiederum auf die „exzentrische Positionalität“ als Konstante menschlichen Daseins insistiert, ${ }^{74}$ so werden nun auch diese Sinnpotentiale zum Problem und schließlich gegen den Akteur selbst gewendet.

Aber auch das ist alles andere als verwunderlich: Denn eine solche negative Anthropologie sucht weder nach Universalien der menschlichen Existenz, noch verwirft sie deren Möglichkeit, sondern übersetzt die Frage nach der Natur des Menschen in die praktisch-funktionale Aufgabe, menschlicher Defizite durch rechtliche, sozialpolitische oder polizeiliche Maßahmen oder durch Strategien der Selbstführung und -disziplinierung zu verhindern, jedenfalls aber zu minimieren. ${ }^{75}$ Weil Risiken aber, anders als entstandene Schäden oder erlittenes Unrecht, nur probabilistisch, also in einem Wahr-

71 Von der Diskussion um so genannte „Schläfer“ ganz zu schweigen.

72 Vgl. hier die Schrift: Idee zu einer allgemeinen Geschichte in weltbürgerlicher Absicht, GS Berlin 1902 ff., ND 1977 (1784), AA VIII, S. 23.

73 Der Mensch. Seine Natur und seine Stellung in der Welt, Berlin 1940, S. 86 und öfter.

74 Die Stufen des Organischen und der Mensch, Berlin 1975, S. 292.

75 Bröckling (Fn. 4), S. 38 ff.. 
scheinlichkeitskalkül, erfassbar sind, generalisiert der präventive Blick den Verdacht und sucht Indizien aufzuspüren, die auf künftige Übel, also Konflikte hindeuten und an denen die vorbeugenden Maßnahmen ansetzen können. ${ }^{76}$ Fluchtpunkt dieser Strategie ist das Ideal der totalen Transparenz. Daraus folgt eine Praxis fortwährender Visualisierung, die wiederum kontinuierliche Kommunikation und Vernetzung erfordert. Ihr hat sich jegliche Form der Risiko- und Konfliktbekämpfung unterzuordnen.

Vor allem für das (positive) Recht und insbesondere für das Strafrecht bedeutet das eine völlige Entgrenzung des Verantwortungszuweisungsprogramms. Entsprechende Unschärfen bei der Bestimmung tatbestandlicher Verletzungsformen werden als Beurteilungsspielräume des Gesetzgebers oder zeitangemessener Kriminalpolitik ausgegeben und so die Differenz zwischen Schuldzurechnung, Strafzumessung und Gefahrenabwehr überspielt. ${ }^{77}$ Welche Schwierigkeiten, aber auch Unwägbarkeiten diese Form der Funktionalisierung mit sich bringen kann, zeigen die Entwicklungen der Vorfeldkriminalisierung im Allgemeinen und der Maßregeln oder Gefährdungsdelikte im Besonderen. Der hier zentrale Rechtsgüterschutz, als Konzept ursprünglich entwickelt, um die Freiheitsräume des Einzelnen gegenüber den Straf- resp. Interventionsanspruch des Staates zu sichern, droht nunmehr, man denke etwa nur an die Novellierung der $\S \S 129$, 129 a, 129 b, 130 StGB, zur gesetzgeberischen wie dogmatischen „Allzweckwaffe“ vermeintlicher oder tatsächlicher Gefahrenbekämpfung zu werden. ${ }^{78}$ Nicht weniger deutlich wird es im Strafprozessrecht, und dort vor allem bei der Implementierung polizeilicher Gefahren- und Verdachtsargumentationen; man denke nur an die Haftgründe, insbesondere an $\S 112$ a StPO, die Telekommunikationsüberwachung ( $§ 100$ a ff. StPO) oder, aktuell, an die Neufassung des BKA-Gesetzes. ${ }^{79}$

76 Das Modell einer negativen Anthropologie ist freilich nicht neu (es wirft vor allem die Frage auf, inwieweit moderne Präventionsargumente wieder mittel- oder unmittelbar an hobbesschen oder ähnlichen Konzepten anknüpfen - und was das im Einzelnen bedeutet oder bedeuten kann). Bereits das 17. Jahrhundert kennt die „Logik des Extrems“ und damit den Versuch, umfassende (Selbst-)Disziplinierungsstrategien zu installieren: exponiert vorgetragen wird das schon von dem Jesuiten Gracian. Letzterer hatte in seinem Handorakel von 1647 nicht nur eine konzise ausgearbeitete Klugheitskonzeption, sondern auch eine entsprechende Verhaltenslehre entwickelt; vgl. dazu etwa H. Lethen, Verhaltenslehren der Kälte, Frankfurt a.M. 1994, S. 53.

77 So zuletzt sogar das Bundesverfassungsgericht E 120, 224 in seiner „Inzestentscheidung“; siehe dazu bereits die Ausführungen im Abschnitt III.2.

78 Siehe inzwischen auch die Regelungen der $\S \S 89$ a, 89 b, 91 StGB. Vgl. dazu die immer noch instruktive Monographie von K. Amelung, Rechtsgüterschutz und Schutz der Gesellschaft, Frankfurt a.M. 1972; darüber hinaus die Arbeit von W. Hassemer, Theorie und Soziologie des Verbrechens, Frankfurt a.M. 1973; ders., NStZ 1989, S. 553 ff. und den von R. Hefendehl et al. herausgegebenen Sammelband, Die Rechtsgutstheorie. Legitimationsbasis des Strafrechts oder dogmatisches Glasperlenspiel, Baden-Baden 2003.

79 Die „Folterdebatte“ der letzten Jahre sei hier nur am Rande erwähnt, siehe aber die Beiträge bei Nitschke (Hrsg.), Rettungsfolter im modernen Rechtstaat? Bochum 2005. - Zur Ausweitung präventions- und gefahrentheoretischer Argumentationen im Straf(verfahrens)recht $R$. Hefendehl, Grenzenlose Vorverlagerung des Strafrechts?, Berlin 2010; G. Jakobs, in: ZStW 97 (1985), S. 751 ff.; E. Weßlau, Vorfeldkriminalisierungen, Berlin 1989, S. 25 ff. und öfter; Wohlers (Fn. 48), S. 110 ff. und schließlich Verf. (Fn. 27), S. 194 ff. und öfter; insbesondere zum BKA-Gesetz BGBl. I v. 25.12. 2008, 3083. 
Wenn sich aber die Verbrechensprävention nicht allein darauf beschränkt, Täter zu bestrafen, um diese, nach heute überwiegender Überzeugung auch andere Gesellschaftsmitglieder, von zukünftigen Straftaten abzuhalten, sondern zugleich Kriminalitätsraten oder Dunkelziffern berechnet und kriminogene Faktoren, wie z.B. Habitualisierungsstrukturen, herauspräpariert, um dann auf diese einzuwirken, so überlagern normalistische Steuerungsmechanismen die normativen Regelungen. ${ }^{80}$ Umgekehrt wird die Normalität selbst normativ aufgeladen. Das Mittel- wird zum Mindestmass, während Abweichungen vom Durchschnitt Risiko- bzw. Tätergruppen definieren und so neuen Präventionsbedarf signalisieren. Die Anziehungskraft (kriminal-)präventiven Handelns beruht insofern vor allem darauf, dass sie die Denormalisierungsangst, möglicherweise die Grundangst der Moderne, mobilisiert, aber eben auch zu bewältigen verspricht. 81

\section{Perspektiven und Schluss}

Die Steuerungs- und Interventionslogik moderner Gesellschaften ist Ausdruck eines gewandelten Legitimationsverständnisses. Risiken und insoweit verbundene Konflikte werden im Kontext systembedingter Kontingenzerwartungen verhandelt und so eine mehr oder weniger - permanent bestehende Bedrohungsmatrix behauptet. In Kauf genommen wird damit ein enormer Aktivismus, der selbst keine Wirklichkeit beschreibt, sondern nur Wahrnehmungen zu eigenen „Wirklichkeiten“ konstruiert: Je mehr von Sicherheit die Rede ist, desto größer ist die reale oder eben imaginierte Unsicherheit, desto dringlicher der Impuls, etwas dagegen tun zu müssen, und desto gravierender die Maßnahmen, die zu diesem Zweck erforderlich und gerechtfertigt erscheinen (Bröckling). Das aber bedeutet, wie bereits betont, die völlige Entgrenzung staatlicher Disziplinierungs- und Fürsorgepraktiken, des (Straf-)Rechts und nicht zuletzt der Kriminalpolitik. Will man eine solche Entgrenzung sozialer Praktiken, vor allem des Rechts, wirksam verhindern oder zumindest normativ einhegen, zugleich aber auf den Konfliktpräventionsgedanken nicht verzichten, so muss das Sicherheitsdispositiv des modernen Staates auf ein Konzept jenseits der hobbesschen Perspektive zurückgeführt werden; auf ein Konzept, dessen politische und rechtliche Grammatik, bei aller Katastrophen- und Denormalisierungsangst, das Autonomisierungspotential einer Gesellschaft und die Rationalitätsstandards individueller Entscheidungen tatsächlich ernst nimmt. Das Aufklärungs- und Rationalisierungskonzept des neuzeitlich-modernen Gewährleistungsmodells muss also selbst wieder reflektiert und insofern rationalisiert werden. Damit wird nicht der Weg in eine staatstheoretische Utopie angetreten oder einer neuen „Metaphysik des Rechts“ das Wort geredet, sondern darauf insistiert, dass auch gegenwärtige, komplex organisierte Gesellschaften auf ein personal vermitteltes - und deshalb immer auch problematisches - Vernunftfundament, modern gesprochen,

80 Dazu Link (Fn. 60), S. 127, 424; in diese Richtung auch Bröckling (Fn. 4), S. 38 ff.

81 Vgl. zu diesem Kontext bereits G. Canguilhem, Das Normale und das Pathologische, München 1974 und H. Popitz, Über die Präventivwirkung des Nichtwissens, Tübingen 1966, S. 18. 
auf gemeinsam generierte Sinnpotentiale, nicht verzichten können; ${ }^{82}$ jedenfalls dann nicht, wenn mit dem allfälligen Sicherheitsargument mehr gemeint sein soll, als eine krude Rechtfertigung macht- und herrschaftsorientierter Normerhaltung. Nur unter dieser Voraussetzung, so scheint es, kann auch das eingangs erwähnte Leistungs-Legitimations-Paradigma noch seine rechts- und sozialstaatliche Funktion erfüllen und schließlich zu dem beitragen, was wir in der Praxis eines funktionierenden Gemeinwesens mit dem Begriff der condition humaine identifizieren.

82 In diesem Sinne bereits E.-W. Böckenförde, Staat, Gesellschaft, Freiheit, Frankfurt a.M. 1976, S. 60. Dort heißt es: „Der freiheitliche, säkularisierte Staat lebt von Voraussetzungen, die er selbst nicht garantieren kann. Das ist das große Wagnis, das er um der Freiheit willen eingegangen ist. Als freiheitlicher Staat kann er einerseits nur bestehen, wenn sich die Freiheit, die er seinen Bürgern gewährt, von innen her, aus der moralischen Substanz des Einzelnen und der Homogenität der Gesellschaft, reguliert. Andererseits kann er diese Regulierungskräfte nicht von sich aus, d.h. mit den Mitteln des Rechtszwangs und autoritativen Gebots zu garantieren versuchen, ohne seine Freiheitlichkeit aufzugeben und - auf säkularisierter Ebene - in jenen Totalitätsanspruch zurückzufallen, aus dem er in den konfessionellen Bürgerkriegen herausgeführt hat.“ 